\title{
Herpes Zoster: An Atypical Cause of Foot Drop - Case Report and Review of the Literature
}

\section{Coogan MK*, Mobley T, Hagemeijer NC, Abbott B, Guss D, Johnson AH and Di Giovanni CW}

Foot \& Ankle Service, Department of Orthopaedic Surgery, Massachusetts General Hospital, Boston, USA

*Corresponding author: Coogan MK, Foot \& Ankle Service, Department of Orthopaedic Surgery, Massachusetts General Hospital, 55 Fruit Street, Boston, Massachusetts 02114-2696, USA

\begin{abstract}
We report on an 81-year-old female who presented with acute, atraumatic right foot drop four weeks after developing herpes zoster in the ipsilateral L4 and L5 dermatomes treated with valacyclovir. Lumbar MRI revealed no explanatory abnormality, and the etiology of her right foot drop was presumed to be due to an outbreak of herpes zoster. After three months of physical therapy and bracing, her right lower extremity weakness greatly improved. We present a comprehensive literature review of atraumatic foot drop pathooetiology, with a focus on herpes zoster as a potential causative agent.
\end{abstract}

\section{Keywords}

Peroneal neuropathy

\section{Introduction}

Herpes zoster, commonly known as shingles, is a viral infection that produces a painful, blistering skin rash. It is caused by reactivation of the dormant varicella zoster virus that resides in the dorsal root ganglia of the central nervous system. The lifetime risk of developing herpes zoster is 20 to $30 \%$, with elderly and immunosuppressed patients being most commonly affected [1]. Timely initiation of antiviral medication may shorten its course, but occasionally neurological complications can persist [2]. Although the nociceptive, sensory, and dermatomal sequellae of active herpes zoster are well known, a relatively uncommon neurological complication is motor paresis of the peroneal nerve which can present clinically as acute foot drop alongside numbness along the dorsum of the foot $[3,4]$.

This report describes an immunologically normal 81-year-old female who developed sudden onset of atraumatic foot drop four weeks after an outbreak of herpes zoster. We provide a comprehensive literature review of the various pathoetiologies of atraumatic foot drop, with a special focus on herpes zoster as a potential causal agent that should be considered in this differential.

\section{Methods}

A comprehensive literature search was conducted using the databases EMBASE, MEDLINE, OviddSP, and PubMed Publisher, with the help of a librarian. The detailed search is presented here:

("Peroneal Neuropathies"[Mesh] OR foot drop[tiab] OR dropfoot[tiab] OR drop foot[tiab] OR dropping foot[tiab] OR peroneal nerve paralysis[tiab] OR peroneal nerve palsy[tiab] OR peroneal nerve dysfunction[tiab] OR peroneal neuropathy[tiab] OR peroneal mononeuropathy[tiab]) AND ("Case-Control Studies"[Mesh] OR "Case Reports"[pt] OR (case*[tiab] AND (report*[tiab] OR stud*[tiab])) OR case*[ti] OR case-control[tiab] OR casecompar*[tiab] OR case stud*[tiab] OR "Observational Study" [Publication Type] OR "Cohort Studies"[Mesh] OR "Retrospective Studies"[Mesh] OR observational[tiab] OR retrospective[tiab] OR cohort[tiab]).

An article was included in the literature search if it had the full text and original data available and was written in English, German, or Dutch. Patients were required to be over the age of 18 with isolated foot drop due to a nontraumatic cause. Articles were excluded if the patient had post-surgical foot drop, post-traumatic foot drop, non-isolated foot drop, or a traumatic herniated disc.

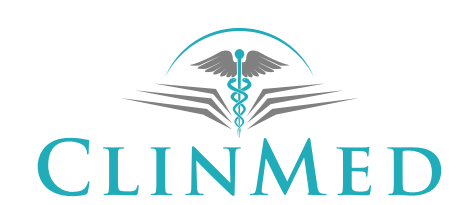

INTERNATIONAL LIBRARY 
Articles were assessed by two independent reviewers (MC, TM) and, upon disagreements, a third reviewer $(\mathrm{NH})$ was utilized in order to reach a consensus.

\section{Case Report}

An 81-year-old female with a medical history of gastroesophageal reflux disease, osteoarthritis, hyperlipidemia, hypertension, and non-radiating chronic low back pain presented to our orthopedic outpatient clinic with right foot drop which had progressively developed roughly four weeks prior to her initial evaluation.

She originally presented to her primary care physician eight weeks prior with burning pain throughout her right lower back and thigh and was initially diagnosed with sciatica. Several days later she returned with a vesicular rash to her proximal lateral right thigh spanning the L4/L5 dermatome. She was then diagnosed with herpes zoster and prescribed a one-week course of valacyclovir. Although the Centers for Disease Control and Prevention (CDC) recommends that adults over the age of 50 receive the herpes zoster vaccine, this patient was unvaccinated. One month after completion of the antiviral treatment, the patient reported progressive "weakness and cramping" and the inability to dorsiflex her right foot. She then suffered an inversion/planterflexion injury to her right ankle while walking in her home and presented to our office for evaluation roughly one week later. She denied any lower extremity motor weakness prior to the herpes zoster outbreak, nor had her pre-existing low back pain ever included radiating symptoms.

On physical examination, she was a well appearing female with normal lower extremity alignment to her right hip, knee and ankle. The previously described vesicular rash to the low back and lateral thigh had resolved. She had mild swelling and tenderness to palpation on the dorsum of her right ankle due to her prior sprain. Her ankle was stable to anterior drawer and talar tilt testing. She has normal passive motion but weakness with active motion of the ankle: dorsiflexion $0 / 5$; plantarflexion $3 / 5$; inversion $3 / 5$; eversion $2 / 5$. She was prescribed a carbon fiber ankle-foot orthosis, physical therapy, in addition to a neurology referral.

Neurologic assessment confirmed the diagnosis of right common peroneal neuropathy alongside a less significantly involved tibial nerve. A lumbar spine MRI, low back and right hip radiographs obtained did not reveal any structural abnormalities or polyradiculopathy that could explain the right foot drop. Based on the delayed neurologic symptoms that appeared weeks after the vesicular rash, it was determined that her foot drop was a likely sequela of the preceding herpes zoster infection. Neither nerve conduction studies nor EMG testing were performed.
After 5 months of physical therapy, the patient reported an improvement in active dorsiflexion strength.

\section{Discussion}

Postherpetic motor paralysis is rare. When it does occur, however, it will generally correlate with the dermatomal distribution of the associated herpes zoster rash $[5,6]$. It is estimated that such segmental zoster paralysis occurs in $0.5-5 \%$ of all herpes zoster cases [5]. Approximately half of such cases involve the cranial nerves, with patients typically demonstrating facial and optic nerve impairment [7]. Peripheral motor involvement affecting distal muscle groups is less common and, when present, is twice as likely to affect the upper extremities as compared to the lower extremities [3].

After varicella exposure, the herpes virus remains dormant in the dorsal root ganglion, a primarily sensory nerve body. The mechanism by which the herpes zoster virus subsequently invades motor neurons is unknown, but it is hypothesized that inflammation produced by the virus can extend from the dorsal root ganglion into the anterior horn (ventral root). The resultant hypervascularity in the perineural tissue, alongside potential disruption of the blood-nerve barrier, may lead to the motor deficits seen clinically $[3,8,9]$.

A literature review revealed numerous causes of isolated atraumatic foot drop, including ganglion cysts of the peroneal nerve [10] , brain lesions [11] , spinal lesions [12] , herniated discs [13] , osteochondromas of the tibia or fibula [14] , autoimmune diseases [15] , and viral infections [5]. Within this search, we identified 11 reported cases of herpes zoster infection precipitating an isolated foot drop [5,9,16-23] . Among these patients, the mean age at time of presentation was 68-yearsold. Seven patients who developed foot drop were immunocompromised $[5,9,16,17,19-21]$, two patients were previously healthy $[22,23]$, and two patients had an unknown medical history $[18,23]$.

Nine patients experienced segmental zoster paralysis due to a herpes zoster rash in the L5 or S1 dermatome 9 , $17-23$, analogous to our patient whose rash occurred in the $L 4 / L 5$ dermatome.

There were two rare cases, however, of nonsegmental zoster paresis wherein patients presented with a rash in the T11 dermatome and a subsequent concomitant foot drop $[5,16]$. In regards to the temporal correlation of these symptoms, ten patients experienced foot drop 1-6 weeks after the herpes zoster diagnosis $[9,16-23]$ and only one case documented onset of foot drop over ten weeks after initial herpes zoster diagnosis [5]. Diagnosis was based upon clinical presentation of vesicular rash temporally associated with a subsequent foot drop. MRI and nerve conduction studies were useful only to exclude other pathology while confirming 
the involvement of the common peroneal nerve.

In the cases reviewed, the majority of patients were treated in similar fashion with an antiviral drug, such as acyclovir or valacyclovir, a brace or splint, and physical therapy. After an average follow-up of 4.3 months, most patients reported almost a full recovery of function $[7,17]$. Approximately half of such patients, however, continued to experience some residual pain and/or weakness [5,9,17,19-21].

\section{Conclusion}

In rare cases, herpes zoster infection can lead to motor deficit in the form of an atypical foot drop-most commonly affecting elderly and immunocompromised patients. In the absence of pre-existent trauma, the presence of foot drop along with concomitant complaints of zoster associated pain and rash in corresponding lumbar dermatomes should suggest the possibility that viral inflammation of the nervous system could account for these findings. This index of suspicion would be helpful in facilitating prompt diagnosis and appropriate non-operative treatment of this patient population.

\section{Funding}

This research did not receive any specific grant from funding agencies in the public, commercial, or not-forprofit sectors.

\section{References}

1. CDC (2008) Prevention of herpes zoster: Recommendations of the Advisory Committee on Immunization Practices (ACIP). MMWR Recomm Rep 57: 1-30.

2. Takahama H, Tsukahara N, Hirayama M, Ito S, Sakuramoto C (2007) Isolated double herpes zoster paresis involving the left facial nerve and the right peroneal nerve following disseminated herpes zoster. J Dermatol 34: 349-352.

3. Helfgott SM, Picard DA, Cook JS (1993) Herpes zoster radiculopathy. Spine (Phila Pa 1976) 18: 2523-2524.

4. Stewart JD (2008) Foot drop: Where, why and what to do? Pract Neurol 8: 158-169.

5. Seo DH, Lee SJ, Hyun JK, Kim TU (2012) A Case of Herpes zoster peripheral polyneuropathy manifested by foot drop in chronic myeloid leukemia. Ann Rehabil Med 36: 724-728.

6. Kawajiri S, Tani M, Noda K, Fujishima K, Hattori N, et al. (2007) Segmental zoster paresis of limbs: Report of three cases and review of literature. Neurologist 13: 313-317.

7. Molinero J, Nagore E, Obon L, Miquel FJ, Aliaga A (2002) Metameric motor paresis following abdominal herpes zoster. Cutis 69: 143-144.
8. Yoleri O, Olmes N, Oztura I, Sengul I, Gunaydin R, et al. (2005) Segmental zoster paresis of the upper extremity: A case report. Arch Phys Med Rehabil 86: 1492-1494.

9. Conliffe TD, Dholakia M, Broyer Z (2009) Herpes zoster radiculopathy treated with fluoroscopically-guided selective nerve root injection. Pain Physician 12: 851-853.

10. Alsahhaf A, Renno WM (2016) Ganglion cyst at the proximal tibiofibular joint in a patient with painless foot drop. Pain Physician 19: 1147-1160.

11. Paliwal VK, Malhotra HS, Sharma R, Shukla R (2008) Delayed diagnosis of brain tumor in a patient with flexor spasms and spastic foot drop. Ann Indian Acad Neurol 11: 254-256.

12. Miwa T, Iwasaki M, Miyauchi A, Okuda S, Oda T (2011) Foot drop caused by a lesion in the thoracolumbar spine. $J$ Spinal Disorders Tech 24: 21-25.

13. Kertmen H, Gurer B, Yimaz ER, Sekerci Z (2015) Acute bilateral isolated foot drop: Report of two cases. Asian J Neurosurg 10: 123-125.

14. Cinar A, Yumrukcal F, Salduz A, Dirik Y, Eralp L (2014) A rare case of 'drop foot' in the pediatric age group: Proximal fibular osteochondroma a report of 5 cases. Int J Surg Case Rep 5: 1068-1071.

15. Basak RB, Narchi H, Bakir M, Joshi S, Conca W (2011) Churg-strauss syndrome without respiratory symptoms in a child. Indian J Dermatol 56: 84-86.

16. Damodaram S, Patel A, Kudva G, Iyadurai S (2012) Foot drop and urinary retention following disseminated varicella zoster infection. Neurology 78.

17. Goodfriend K, Eickmeyer SM (2012) Foot drop due to herpes zoster after treatment for lymphoma: A case report. PM\&R 4: S258.

18. Gopal KVT, Sarvani D, Raju PVK, Rao GR, Venkateswarlu K (2010) Herpes zoster motor neuropathy: A clinical and electrophysiological study. Indian Journal of Dermatology, Venereology and Leprology 76: 569-571.

19. Kohan K, Deluca H (2012) Painful foot drop secondary to Herpes zoster infection. University of Cincinnati.

20. Leo AM, Kasper DA, Saxena A (2009) Atypical herpes zoster infection preceded by sciatica foot drop. Arch Dermatol 145: 954-955.

21. Mirdamadi A, Kao C (2015) Unusual case of motor neuropathy secondary to herpes zoster: Case report. PM\&R 7: S164.

22. Sachs GM (1996) Segmental zoster paresis: An electrophysiological study. Muscle Nerve 19: 784-786.

23. Sprenger De Rover WB, Alazzawi S, Hallam PJ, Hutchinson R, Di Mascio L (2011) Herpes zoster virus: An unusual but potentially treatable cause of sciatica and foot drop. Orthopedics 34: 965-968. 